\title{
Lower bound on inelasticity in pion-pion scattering
}

\author{
André Martin ${ }^{1, *}$ and S. M. Roy ${ }^{2, \dagger}$ \\ ${ }^{1}$ Theoretical Physics Division, CERN, CH-1211 Geneva 23, Switzerland \\ ${ }^{2}$ HBCSE, Tata Institute of Fundamental Research, Mumbai 400 088, India
}

(Received 20 October 2017; published 14 December 2017)

\begin{abstract}
Assuming that the pion-pion scattering amplitude and its absorptive part are analytic inside an ellipse in the complex $t$ plane with foci $t=0, u=0$ and right extremity $t=4 m_{\pi}^{2}+\epsilon,(\epsilon>0)$ - except for cuts prescribed by the Mandelstam representation for $t \geq 4 m_{\pi}^{2}, u \geq 4 m_{\pi}^{2}$, and bounded by $s^{N}$ on the boundary of this domain-we prove that for $s \rightarrow \infty, \sigma_{\text {inel }}(s)>\frac{\text { const }}{s^{5 / 2}} \exp \left[-\frac{\sqrt{s}}{4}(N+5 / 2) \ln s\right]$.
\end{abstract}

DOI: 10.1103/PhysRevD.96.114014

\section{INTRODUCTION}

It is well known that if there is no inelasticity, the scattering amplitude must be zero. However, there is no quantitative estimate of the amount of inelasticity required. This is what we try to do. There are various proofs of the fact that the scattering amplitude must be zero if there is no inelasticity. A very appealing attempt has been made by Cheung and Toll [1]. Their idea is to repeatedly use elastic unitarity at all energies to the point where an absurd analyticity domain emerges that is much too large. However, even after the enlargement of the pion-pion analyticity domain by one of us in 1966 [2], it is not obvious that they have really succeeded. Dragt [3] proposed a proof that is nice but not quite complete: it uses the fact that partial-wave amplitudes for very large angular momenta are dominated by the nearest singularities in the crossed channel. More analyticity is required than what has been proved from field theory [2]. For instance, the Mandelstam representation [4] with a finite number of subtractions is largely sufficient. In fact, we require much less than that. Since we shall also use the dominance of the nearest singularities for large angular momenta, we state at the same time the assumption he needs and our assumption. If we use the standard Mandelstam variables $s, t, u$ and choose units such that the pion mass $m_{\pi}=1$, we need fixed-energy analyticity in an ellipse with foci at $t=0$ and $u=0$ and right extremity at $t=4+\epsilon$, minus the obvious cuts $t \geq 4, u \geq 4$ for the amplitude, and $t \geq 4+64$ / $(s-16), \quad u \geq 4+64 /(s-16)$ for the absorptive part (see Fig. 1). From field theory we only get, for the absorptive part, an ellipse with right extremity at $t=4$ exactly, and for the amplitude a region containing $|t|<4$.

\footnotetext{
martina@mail.cern.ch

‘smroy@hbcse.tifr.res.in
}

Published by the American Physical Society under the terms of the Creative Commons Attribution 4.0 International license. Further distribution of this work must maintain attribution to the author(s) and the published article's title, journal citation, and DOI.
In fact, for $|t|<4$ fixed- $t$ dispersion relations are valid, and with our assumptions they are valid for $|t|<4+\epsilon$. With these assumptions we can prove that there must be inelasticity at energies such that $s>16+64 / \epsilon$. For instance, if $\epsilon=12$ (corresponding to the full $t$-channel elastic strip), we must have inelasticity for $s>22$.

For simplicity, we look first at the $\pi^{0} \pi^{0}$ scattering amplitude $F(s, t)$, where $\pi^{0}$ is a fictitious isospin-zero neutral pseudoscalar particle. It has the partial-wave expansion

$$
\begin{aligned}
& F(s, t)=\sum_{l=0}^{\infty}(2 l+1) f_{l}(s) P_{l}\left(1+\frac{2 t}{s-4}\right), \\
& f_{l}(s)=a_{l}(s) / \rho(s) ; \quad \rho(s) \equiv \frac{2 k}{\sqrt{s}}=\sqrt{\frac{s-4}{s}},
\end{aligned}
$$

with the unitarity constraint

$$
\begin{array}{ll}
\operatorname{Im} a_{l}(s)=\left|a_{l}(s)\right|^{2}, & 4 \leq s \leq 16, \\
\operatorname{Im} a_{l}(s) \geq\left|a_{l}(s)\right|^{2}, & s \geq 16 .
\end{array}
$$

The optical theorem gives

$$
\sigma_{\mathrm{tot}}=\frac{8 \pi}{k^{2}} \sum_{l=0}^{\infty}(2 l+1) \operatorname{Im} a_{l}(s)=\frac{16 \pi}{k \sqrt{s}} F_{s}(s, 0)
$$

where $F_{s}(s, t)$ denotes the $s$-channel absorptive part $\operatorname{Im} F(s, t)$. Similar unitarity conditions hold in the $t$ and $u$ channels. The normalization specified by the above choice of $\rho(s)$ corresponds to $F(4,0)=S$-wave scattering length $a_{0}$. For the generalization to real pions of isospin 1, we shall use the same normalizations as above, with $F(s, t), f_{l}(s), a_{l}(s)$, $\sigma_{\text {tot }}, A_{s}(s, 0), F(4,0), a_{0}$ being replaced by the corresponding quantities with a superscript $I$, e.g., $F^{I}(s, t), ., a_{0}^{I}$, respectively.

Our strategy will be the following. We write the partialwave amplitudes as well as their imaginary parts as contour integrals along the ellipse mentioned above, and add the contribution of the cuts (see Fig. 1). Then we try to get an 


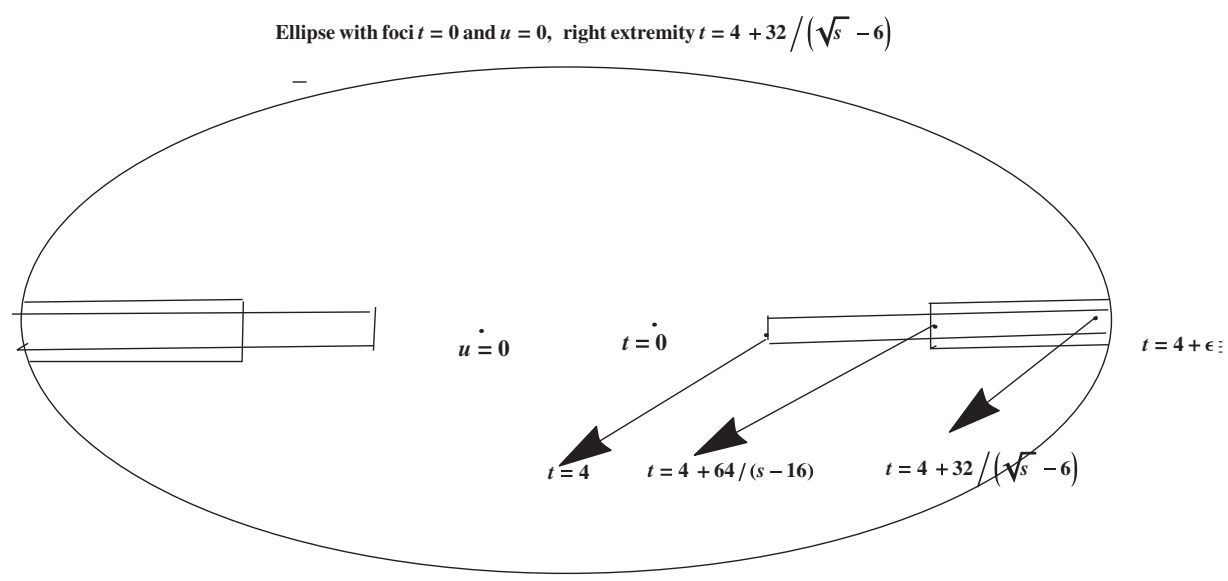

FIG. 1. The amplitude $F(s, t)$ is assumed to be analytic in $t$ within the ellipse shown except for cuts $t \geq 4, u \geq 4$; its absorptive part $F_{s}(s, t)$ for $s \geq 20$ is assumed to be analytic in $t$ within the same ellipse except for cuts for $t \geq 4+\frac{64}{s-16}, u \geq 4+\frac{64}{s-16}$. The truncated Froissart-Gribov formulas for $f_{l}(s)$ [Eq. (20)] and $\operatorname{Im} f_{l}(s)$ [Eq. (21)] follow from this. Note that the horizontal and vertical scales in this figure are not the same.

upper bound on the partial-wave amplitude $f_{l}$ for which we need an upper bound $B(s)$ on the whole ellipse. We also seek a lower bound on its imaginary part $\operatorname{Im} f_{l}$, for which we need a bound on the discontinuity of the absorptive part which is nothing but the Mandelstam double spectral function. In fact, this is what was missing in the work of Dragt [3]. This will be done in the next section.

\section{A. Domain of positivity of the double spectral function and a lower bound}

First, we recall the results of Mahoux and one of us [5] on the domain of positivity of the double spectral function. For $s>20$, the absorptive part in the $s$-channel has a cut beginning at

$$
t=4+\frac{64}{s-16}
$$

From $t=4$ to $t=4+\epsilon<16$, the discontinuity across the cut is given by the Mandelstam form of the $t$-channel elastic unitarity condition on one of the double spectral functions $\rho_{s t}(s, t)$,

$\rho_{s t}(s, t)=\frac{2 \rho(t)}{\pi} \iint \frac{d z_{1} d z_{2}}{\sqrt{H\left(z, z_{1}, z_{2}\right)}} F_{s}\left(s_{1}, t\right) F_{s}\left(s_{2}, t\right)^{*}$,

where

$$
\begin{aligned}
\rho(t) & =\sqrt{\frac{t-4}{t}}, \quad z=1+\frac{2 s}{t-4}, \quad z_{0} \equiv 1+\frac{8}{t-4}, \\
z_{i} & =1+\left(2 s_{i}\right) /(t-4), \quad i=1,2,
\end{aligned}
$$

and

$$
\begin{aligned}
H\left(z, z_{1}, z_{2}\right) & =z^{2}+z_{1}^{2}+z_{2}^{2}-1-2 z z_{1} z_{2} \\
& =\left(z-z_{+}\right)\left(z-z_{-}\right),
\end{aligned}
$$

with

$$
z_{ \pm}=z_{1} z_{2} \pm \sqrt{\left(z_{1}^{2}-1\right)\left(z_{2}^{2}-1\right)}
$$

The domain of integration in the $z_{1}-z_{2}$ plane is bounded by the three lines

$$
z_{1}>z_{0}, \quad z_{2}>z_{0}, \quad z>z_{+} \cdot
$$

If we define

$$
z=\cosh \theta ; \quad z_{i}=\cosh \theta_{i}, \quad i=0,1,2,
$$

then the region (9) becomes just a triangle in the $\theta_{1}-\theta_{2}$ plane bounded by the lines (see Fig. 2)

$$
\theta_{0} \leq \theta_{1}, \quad \theta_{0} \leq \theta_{2}, \quad \theta_{1}+\theta_{2} \leq \theta
$$

These inequalities imply that for $i=1,2, \theta_{0} \leq \theta_{i} \leq \theta-\theta_{0}$, i.e.,

$$
z_{0} \leq z_{i} \leq z z_{0}-\sqrt{\left(z^{2}-1\right)\left(z_{0}^{2}-1\right)}
$$

They also imply that $\theta \geq 2 \theta_{0}$, which gives the boundary curve of the spectral region

$$
s \geq \frac{16 t}{t-4}
$$

It will be crucial to recall the observation of Mahoux and Martin [5] that when $\theta \leq 3 \theta_{0}$, the inequalities (11) imply 


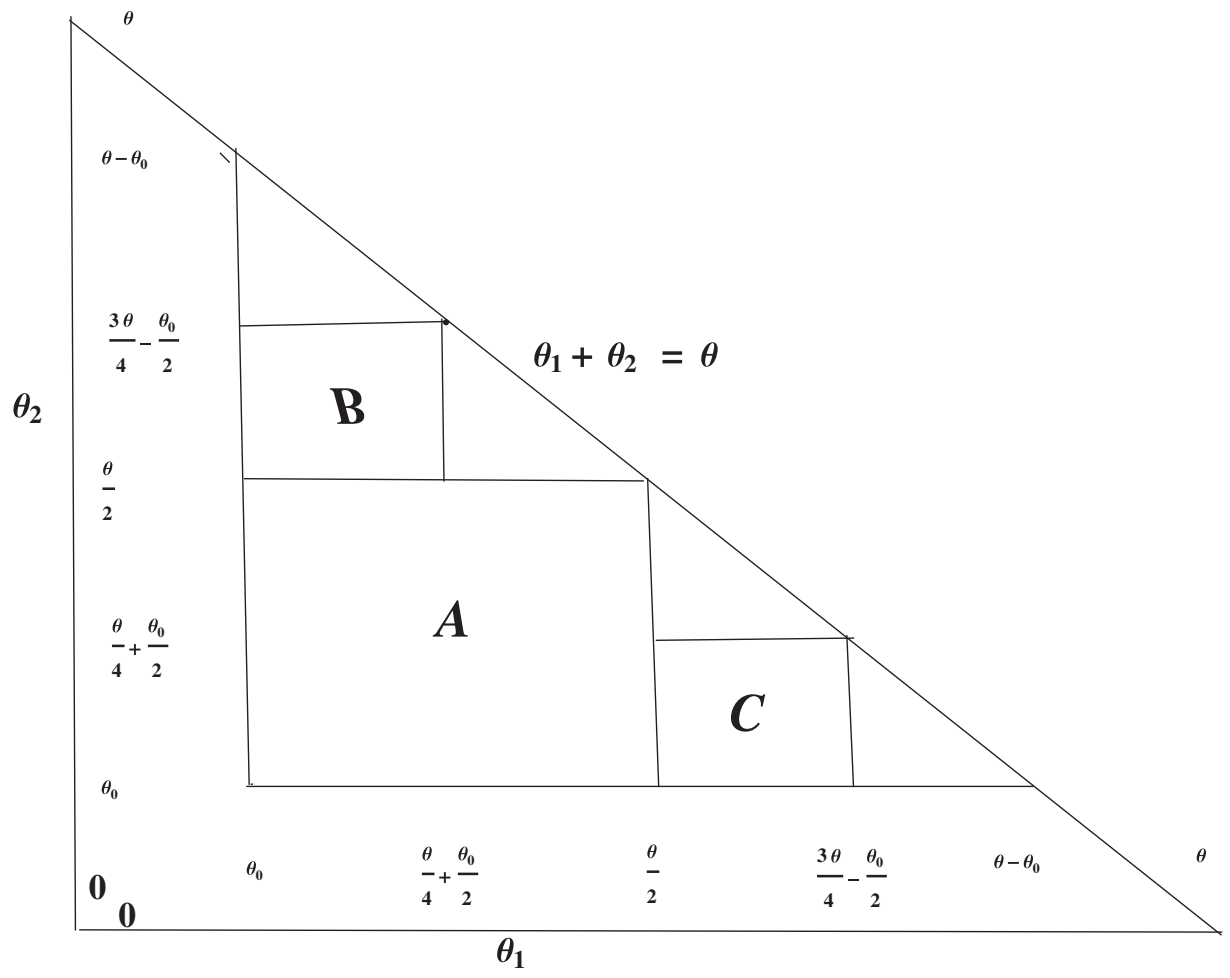

FIG. 2. We show the triangular integration region in the $\theta_{1}-\theta_{2}$ plane in Mandelstam's continued elastic unitarity equation in the $t$ channel, defined by $\theta_{1} \geq \theta_{0}, \theta_{2} \geq \theta_{0}$, and $\theta_{1}+\theta_{2} \leq \theta$. The subregions $A, B, C$ are used to calculate lower bounds on the double spectral function.

that only values of $\theta_{i} \leq 2 \theta_{0}$ for $i=1$, 2, i.e., only values of $F_{s}\left(s_{i}, t\right)$ outside the spectral region for $i=1,2$ are needed to compute the double spectral function. In this region, the convergent partial-wave expansion

$F_{s}\left(s_{i}, t\right)=\sum_{l=0}^{\infty}(2 l+1) \operatorname{Im} f_{l}\left(s_{i}\right) P_{l}\left(1+\frac{2 t}{s_{i}-4}\right), \quad i=1,2$,

the positivity of $\operatorname{Im} f_{l}\left(s_{i}\right)$, and the inequalities $P_{l}(1+2 t /$ $\left.\left(s_{i}-4\right)\right)>1$ imply that $F_{s}\left(s_{i}, t\right)>0$ for $i=1,2$. Hence, the double spectral function $\rho_{s t}(s, t)$ is positive when $\theta \leq 3 \theta_{0}$, i.e., for

$$
\begin{aligned}
& 4 \leq t \leq 16, \quad \text { and } \quad \frac{16 t}{t-4} \leq s \leq 4\left(\frac{3 t+4}{t-4}\right)^{2}, \\
& \text { i.e., } 4+64 /(s-16) \leq t \leq 4+32 /(\sqrt{s}-6)
\end{aligned}
$$

Since $\rho(s, t)$ is symmetrical in its arguments, it is also positive for

$$
4 \leq s \leq 16, \quad \frac{16 s}{s-4} \leq t \leq 4\left(\frac{3 s+4}{s-4}\right)^{2}
$$

\section{LOWER BOUND ON INELASTICITY}

We shall now obtain a lower bound on $\rho(s, t)$ in the domain (15) in terms of the total cross sections $\sigma_{\text {tot }}\left(s_{1}\right)$, $\sigma_{\text {tot }}\left(s_{2}\right)$, where $s_{1}, s_{2}$ are such that Eq. (12) holds for the corresponding $z_{1}, z_{2}$. We then deduce a lower bound on inelasticity. It will then follow that if there is no inelasticity at one (and only one) energy in the $s$-channel $(s>20)$, the double spectral function must vanish in the range $t=$ $4+64 /(s-16)$ to $t=4+32 /(\sqrt{s}-6)$, and hence there is an interval of energy given by Eq. (12) in which the total cross section vanishes. This is impossible, and hence the scattering amplitude is zero. It must be realized that only a small fraction of the Mandelstam representation is used.

Now, the question posed to one of us by Miguel F. Paulos (during a conference organized by João Penedones at EPFL, Lausanne) was whether the inelastic cross section could be arbitrarily small. We want to show that-with some assumptions that are much weaker than the Mandelstam representation, but slightly stronger than what has been proved from local field theory-there exists a lower bound to inelasticity,

$$
\sigma_{\text {inelastic }}>C \exp \left(-\sqrt{\left(s / s_{0}\right)} \log \left(s / s_{0}\right)\right) \text {. }
$$

The strategy we shall use is based on the results of Mahoux and Martin [5] on the positivity of double spectral functions, and on the research of Dragt [3], viz., that the 
real and imaginary parts of the partial-wave amplitudes are dominated by the contributions of the nearby cuts in the crossed channel:

from $t=4$ to $t=t_{M}(s) \quad$ for $\operatorname{Re} f_{l}$ and $f_{l}, \quad$ and from $t=4+\frac{64}{s-16}$ to $t=t_{M}(s)$ for $\operatorname{Im} f_{l}$,

where

$$
t_{M}(s) \equiv 4+\frac{32}{\sqrt{s}-6} .
$$

\section{A. Estimates of $f_{l}(s)$ and $\operatorname{Im} f_{l}(s)$}

We shall use a truncated Froissart-Gribov representation for $\operatorname{Re} f_{l}(s)$ and $\operatorname{Im} f_{l}(s)$. It follows from the analyticity of $F(s, t)$ in $t$ within an ellipse with right extremity $t=t_{M}(s)$ and foci $t=0$ and $u=0$, except for the cuts $4 \leq t \leq t_{M}(s)$ and $4 \leq u \leq t_{M}(s)$. For $l$ even,

$$
\begin{aligned}
f_{l}(s)= & \frac{1}{\pi k^{2}} \int_{4}^{4+\frac{32}{\sqrt{s}-6}} Q_{l}\left(1+\frac{2 t}{s-4}\right) F_{t}(s, t) d t \\
& +\frac{1}{4 i \pi k^{2}} \int_{\Gamma} Q_{l}\left(1+\frac{2 t}{s-4}\right) F(s, t) d t,
\end{aligned}
$$

where $\Gamma$ is an ellipse with foci at $t=0$ and $u=0$, and right extremity at $t=4+\frac{32}{\sqrt{s}-6}$ (see Fig. 1).

Hence,

$$
\begin{aligned}
\operatorname{Im} f_{l}(s)= & \frac{1}{\pi k^{2}} \int_{4+\frac{64}{s-16}}^{4+\frac{32}{\sqrt{s-6}}} Q_{l}\left(1+\frac{2 t}{s-4}\right) \rho(s, t) d t \\
& +\frac{1}{4 i \pi k^{2}} \int_{\Gamma} Q_{l}\left(1+\frac{2 t}{s-4}\right) F_{s}(s, t) d t,
\end{aligned}
$$

where $\rho(s, t)$ is given by the Mandelstam equation (5). As noted earlier, if $s$ is in the Mahoux-Martin domain (15), $\rho(s, t)$ is positive.

Now we postulate that $F(s, t)$ and $F_{s}(s, t)$ are bounded by $B(s)$ in the ellipse $\Gamma$. The behavior of $B(s)$ for $s \rightarrow \infty$ will be discussed later. Now we need some estimates on the $Q_{l}$ 's. We prove that, for $z$ real and $>1$ (see the Appendix),

$$
\begin{gathered}
\sqrt{\frac{\pi}{2 l+2}} \frac{1}{\left(z+\sqrt{z^{2}-1}\right)^{l+1}}<Q_{l}(z) \\
<\frac{1}{\left(z+\sqrt{z^{2}-1}\right)^{l}} \frac{1}{2}|\ln | \frac{z+1}{z-1}||
\end{gathered}
$$

and for $z=\cosh \left(\left(\theta_{1}+i \theta_{2}\right)\right)$ (see the Appendix),

$$
\left|Q_{l}\left(\cosh \left(\left(\theta_{1}+i \theta_{2}\right)\right)\right)\right|<\left|Q_{l}\left(\cosh \left(\left(\theta_{1}\right)\right)\right)\right| .
$$

This means that on an ellipse with foci $\cos \theta= \pm 1$ the modulus of $Q_{l} \cos \theta$ is maximum at the right extremity.
We can get a bound on $\left|f_{l}\right|$,

$$
\left|f_{l}\right|<\frac{1}{4 \pi k^{2}} Q_{l}\left(1+\frac{8}{s-4}\right) B(s) L(s),
$$

where $L(s)$ is the perimeter of the ellipse with extremities at

$$
\cos \theta_{s}= \pm\left(1+\frac{1}{2 k^{2}}\left(4+\frac{32}{\sqrt{s}-6}\right)\right)
$$

plus 4 times the length of the cuts $t=4$ to $t=4+\frac{32}{\sqrt{s}-6}$.

For $s>16$,

$$
L(s)<4 s .
$$

Now we need a lower bound for $\operatorname{Im} f_{l}(s) . \operatorname{Im} f_{l}(s)$ is given by a contour integral including the contribution from the cuts and the ellipse. We use the fact that $Q_{l}()$ is a decreasing function for an argument $>1$. We arbitrarily limit the integration on the cuts to

$$
4+\frac{64}{s-16}<t<4+\frac{64+P(s)}{s-16},
$$

where

$P(s)<$ const, $\quad 4+\frac{64+P(s)}{s-16}<4+\frac{32}{\sqrt{s}-6}$,

which is certainly valid for sufficiently large $s$. A lower bound on $\operatorname{Im} f_{l}$ is given by

$$
\begin{aligned}
\operatorname{Im} f_{l}> & \frac{1}{\pi k^{2}} Q_{l}\left(1+\frac{1}{s-4}\left(8+\frac{128+2 P(s)}{s-16}\right)\right) \\
& \times \int_{4+\frac{64}{s-16}}^{4+\frac{64+P(s)}{s-16}} \rho(s, t) d t \\
& -\frac{1}{4 \pi k^{2}} B(s) L(s) Q_{l}\left(1+\frac{1}{s-4}\left(8+\frac{64}{\sqrt{s}-6}\right)\right) .
\end{aligned}
$$

Notice that, according to Ref. [5], $\rho(s, t)$ is strictly positive, as given by the double integral of Mandelstam in the strip $4<t<4+32 /(\sqrt{s}-6)$.

Now, given $B(s), L(s)$, and $\rho(s, t)$, it is possible to prove that $\left|f_{l}\right|^{2}$ is strictly less than $\operatorname{Im} f_{l}$ for sufficiently large $l$. We have

$$
\left|f_{l}\right|^{2}<\frac{1}{\left(4 \pi k^{2}\right)^{2}} Q_{l}^{2}\left(1+\frac{8}{s-4}\right)|B(s)|^{2}|L(s)|^{2},
$$

and so

$$
\begin{aligned}
\frac{\operatorname{Im} f_{l}}{\left|f_{l}\right|^{2}}> & \frac{16 \pi k^{2}}{|B(s)|^{2}|L(s)|^{2}} \frac{Q_{l}\left(x_{1}\right)}{Q_{l}^{2}\left(x_{2}\right)} \times \int_{4+\frac{64}{s-16}}^{4+\frac{64+P(s)}{s-16}} \rho(s, t) d t \\
& -\frac{4 \pi k^{2}}{B(s) L(s)} \frac{Q_{l}\left(x_{3}\right)}{Q_{l}^{2}\left(x_{2}\right)},
\end{aligned}
$$


where we define

$$
\begin{aligned}
& x_{1}=1+\frac{1}{s-4}\left(8+\frac{128+2 P(s)}{s-16}\right), \\
& x_{2}=1+\frac{8}{s-4}, \\
& x_{3}=1+\frac{1}{s-4}\left(8+\frac{64}{\sqrt{s}-6}\right) .
\end{aligned}
$$

It is convenient to denote

$$
\begin{aligned}
& R_{1}=\frac{2 x_{2}^{2}-1+\sqrt{\left(2 x_{2}^{2}-1\right)^{2}-1}}{x_{1}+\sqrt{x_{1}^{2}-1}}, \\
& R_{2}=\frac{x_{3}+\sqrt{x_{3}^{2}-1}}{x_{1}+\sqrt{x_{1}^{2}-1}} .
\end{aligned}
$$

Note that $x_{2}<x_{1}$, and for sufficiently large $s$,

$$
\begin{aligned}
& x_{1}<x_{3} \quad \text { and } \quad x_{1}<2 x_{2}^{2}-1, \\
& \text { and hence } R_{1}>1, \quad R_{2}>1 .
\end{aligned}
$$

We now obtain bounds on the relevant Legendre functions. Using the results (A12) and (A18) from the Appendix, we have

$$
\begin{aligned}
\frac{Q_{l}\left(x_{1}\right)}{Q_{l}^{2}\left(x_{2}\right)} & \geq \frac{1}{2 x_{2} Q_{0}\left(x_{2}\right)} \frac{Q_{l}\left(x_{1}\right)}{Q_{l}\left(2 x_{2}^{2}-1\right)} \\
& \geq \frac{1}{2 x_{2} Q_{0}\left(x_{2}\right)} R_{1}^{l+1} .
\end{aligned}
$$

Further, Eqs. (A18) and (A3) from the Appendix yield

$$
\frac{Q_{l}\left(x_{3}\right)}{Q_{l}^{2}\left(x_{2}\right)} \leq \sqrt{\frac{2(l+1)}{\pi}}\left(\frac{R_{1}}{R_{2}}\right)^{l+1},
$$

and Eq. (A18) gives

$$
\frac{Q_{l}\left(x_{3}\right)}{Q_{l}\left(x_{1}\right)} \leq\left(\frac{1}{R_{2}}\right)^{l+1}
$$

We now have

$$
\begin{aligned}
\frac{\operatorname{Im} f_{l}}{\left|f_{l}\right|^{2}}> & \frac{16 \pi k^{2}}{|B(s)|^{2}|L(s)|^{2}} \frac{1}{2 x_{2} Q_{0}\left(x_{2}\right)} R_{1}^{l+1} \\
& \times \int_{4+\frac{64}{s-16}}^{4+\frac{64+P(s)}{s-16}} \rho(s, t) d t \\
& -\frac{4 \pi k^{2}}{B(s) L(s)} \sqrt{\frac{2(l+1)}{\pi}}\left(\frac{R_{1}}{R_{2}}\right)^{l+1},
\end{aligned}
$$

without asymptotic approximations.
For $s \rightarrow \infty$,

$x_{1}+\sqrt{x_{1}^{2}-1} \sim 1+\frac{4}{\sqrt{s}}+\frac{8}{s}+\cdots$,

$x_{3}+\sqrt{x_{3}^{2}-1} \sim 1+\frac{4}{\sqrt{s}}+\frac{24}{s}+\cdots$,

$2 x_{2}^{2}-1+\sqrt{\left(2 x_{2}^{2}-1\right)^{2}-1} \sim 1+\frac{8}{\sqrt{s}}+\frac{32}{s}+\cdots$,

$R_{1} \sim 1+4 / \sqrt{s}$, and $\left(1 / R_{2}\right) \sim 1-16 / s$

It is clear that since $R_{2}>1$, for large enough $l$, i.e., for

$$
l>L_{0}(s)=\text { const } s \ln s, \quad s \rightarrow \infty,
$$

the contribution of the first term on the right-hand side of Eq. (37) involving a positive double spectral function is dominant, and that term implies that

$$
\frac{\operatorname{Im} f_{l}}{\left|f_{l}\right|^{2}} \rightarrow \infty, \quad l>\text { const } s \ln s .
$$

Hence the inelastic cross section is dominant and nonzero for $l>L_{0}(s)$. The fact that $\rho(s, t)$ is different from zero is essential. We now evaluate the lower bound on $\operatorname{Im} f_{l}$, and hence on the inelastic cross section at high energies.

\section{LOWER BOUND ON THE DOUBLE SPECTRAL FUNCTION}

We must get a lower bound on $\rho(s, t)$. This is relatively easy. We return to the Mandelstam equation (5) for $4<$ $t<16$ and restrict ourselves to the Mahoux-Martin domain (15) of positivity of $\rho(s, t)$. To get a lower bound on $\rho(s, t)$ we shall do rather wild majorizations.

(1) We reduce the domain of integration in the $\theta_{1}-\theta_{2}$ plane (11) to the union of three regions $A, B, C$ (see Fig. 2):

$$
\begin{aligned}
& A: \theta_{0} \leq \theta_{i} \leq \theta_{M} \equiv \theta / 2, \quad i=1,2, \\
& \text { i.e., } z_{0} \leq z_{i} \leq z_{M} \equiv \sqrt{\frac{1+z}{2}}, \quad i=1,2 ; \\
& B: \theta_{0} \leq \theta_{1} \leq \theta_{1 M} \equiv\left(\theta / 4+\theta_{0} / 2\right), \\
& \text { i.e., } z_{0} \leq z_{1} \leq z_{1 M} \equiv \cosh \left(\theta / 4+\theta_{0} / 2\right), \\
& \theta / 2 \leq \theta_{2} \leq \theta_{2 M} \equiv\left(3 \theta / 4-\theta_{0} / 2\right), \\
& \text { i.e., } z_{M} \leq z_{2} \leq z_{2 M} \equiv \cosh \left(3 \theta / 4-\theta_{0} / 2\right) \text {; } \\
& C: \theta_{0} \leq \theta_{2} \leq \theta_{1 M}, \\
& \text { i.e., } z_{0} \leq z_{2} \leq z_{1 M}, \\
& \theta / 2 \leq \theta_{1} \leq \theta_{2 M}, \\
& \text { i.e., } z_{M} \leq z_{1} \leq z_{2 M} .
\end{aligned}
$$


Notice that under $z_{1} \leftrightarrow z_{2}$, the regions $B \leftrightarrow C$ and $A \leftrightarrow A$.

(2) Using Eqs. (7) and (8), we shall replace $H\left(z, z_{1}, z_{2}\right)$ in the denominator by simple upper bounds on it in the three regions:

A: $H\left(z, z_{1}, z_{2}\right) \leq(z-1)^{2}$;

$B, C: H\left(z, z_{1}, z_{2}\right) \leq\left(z-z_{-}\right)^{2} \leq\left(z-z_{3}\right)^{2}$,

$z_{3} \equiv \cosh \left(\theta / 4-\theta_{0} / 2\right)$.

It will be convenient to define

$\left(z_{M}, z_{1 M}, z_{2 M}, z_{3}\right)=1+\frac{2}{t-4}\left(s_{M}, s_{1 M}, s_{2 M}, s_{3}\right)$.

(3) Since we are in the Mahoux-Martin domain in which $F_{s}\left(s_{1}, t\right)$ and $F_{s}\left(s_{2}, t\right)$ have convergent partial-wave expansions with positive partial waves, and $t$ is positive, the absorptive parts obey the bounds

$F_{s}\left(s_{i}, t\right) \geq F_{s}\left(s_{i}, 0\right)=\frac{k_{i} \sqrt{s_{i}}}{16 \pi} \sigma_{\mathrm{tot}}\left(s_{i}\right), \quad i=1,2$.

They also obey stronger bounds in terms of $\sigma_{\text {tot }}\left(s_{i}\right)$ (originally derived by Martin [2]) or $0<t<4$, but they are also valid for $4<t<4+\frac{32}{\sqrt{s}-6}$ under the present assumptions. At high energies they have the simple form

$$
\begin{aligned}
F_{s}\left(s_{i}, t\right) & \geq F_{s}\left(s_{i}, 0\right) \frac{2 I_{1}\left(x_{i}\right)}{x_{i}}\left(1+O\left(1 / \sqrt{s_{i}}\right)\right), \\
x_{i} & =\sqrt{t \sigma_{\mathrm{tot}}\left(s_{i}\right) /(4 \pi)}, \quad i=1,2 .
\end{aligned}
$$

Using the majorizations (1) and (2) and the weaker bound (42) in 3), we obtain

$\rho(s, t) \geq \frac{4}{\pi \sqrt{t(t-4)}}\left[\frac{1}{s} I^{2}\left(s_{M}\right)+\frac{2}{s-s_{3}} I\left(s_{1 M}\right) I\left(s_{2 M}\right)\right]$,

where the first term in the brackets on the right is the contribution of region $A$ and the second term is that of regions $B$ and $C$,

$$
I\left(s_{M}\right) \equiv \int_{4}^{s_{M}} \frac{d s_{1} k_{1} \sqrt{s_{1}} \sigma_{\mathrm{tot}}\left(s_{1}\right)}{16 \pi},
$$

and $I\left(s_{1 M}\right)$ and $I\left(s_{2 M}\right)$ are defined similarly by replacing $s_{M}$ by $s_{1 M}$ and $s_{2 M}$, respectively. Note that $s_{M}, s_{1 M}$, and $s_{2 M}$ depend on $s$, $t$. E.g.,

$$
2 s_{M}=\sqrt{(t-4)(t-4+s)}-(t-4) \text {. }
$$

A simple bound is obtained by retaining only the region $A$. In addition to the above results for general $P(s)$, we shall evaluate bounds on $I\left(s_{M}\right), \rho(s, t)$ and the integral over $t$ of $\rho(s, t)$, for two simple choices of $P(s)$.

(i) $P(s)$ independent of $s$ : Let $P_{1}<p<P_{2}$; then, we can get a lower bound on the integral over $t$ of $\rho(s, t)$ by restricting to the interval

$$
\begin{aligned}
& \left(64+P_{1}\right) /(s-16)<t-4=(64+p) /(s-16) \\
& \quad<\left(64+P_{2}\right) /(s-16) .
\end{aligned}
$$

Then,

$$
t(t-4)<\frac{\left(64+P_{2}\right)\left(4 s+P_{2}\right)}{(s-16)^{2}} .
$$

For a large enough fixed $s, s_{M}$ is an increasing function of $t$, and hence its minimum value is at the lowest value of $t$,

$$
\begin{aligned}
& s_{M} \geq\left(s_{M}\right)_{\min } \\
& \equiv \frac{\sqrt{\left(64+P_{1}\right)\left[64+P_{1}+s(s-16)\right]}-\left(64+P_{1}\right)}{2(s-16)}
\end{aligned}
$$

and

$$
I\left(s_{M}\right) \geq I\left(\left(s_{M}\right)_{\min } \cdot\right.
$$

Finally, we have the bound

$$
\int_{4+\frac{44+P_{1}}{s-16}}^{4+\frac{64+P_{2}}{s-16}} \rho(s, t) d t \geq \frac{4\left(P_{2}-P_{1}\right) I^{2}\left(\left(s_{M}\right) \min \right)}{\pi s \sqrt{\left(64+P_{2}\right)\left(4 s+P_{2}\right)}},
$$

which is positive definite and $>$ const $s^{-3 / 2}$ unless the total cross section vanishes identically at all energies up to $\left(s_{M}\right)_{\min }$.

(ii) $P(s) \rightarrow 0$ for $s \rightarrow \infty$ : In this case, we integrate over the region

$$
\begin{aligned}
4+ & \frac{\left(64+p_{1}(s)\right)}{(s-16)}<t=4+\frac{(64+p(s))}{(s-16)} \\
& <4+\frac{\left(64+p_{2}(s)\right)}{(s-16)},
\end{aligned}
$$

where $p_{1}(s)$ and $p_{2}(s) \rightarrow 0$, for $s \rightarrow \infty$, and we get $s_{M}-4 \sim p(s) / 32 \rightarrow 0$. In the integral defining $I\left(s_{M}\right)$ we can therefore replace 


$$
\sigma_{\text {tot }} \rightarrow 8 \pi a_{0}^{2}
$$

where $a_{0}$ is the $S$-wave scattering length, and obtain

$$
I^{2}\left(s_{M}\right) \rightarrow(p(s) / 32)^{3} a_{0}^{4} / 9 \geq\left(p_{1}(s) / 32\right)^{3} a_{0}^{4} / 9 .
$$

Finally, for $s \rightarrow \infty, p_{1}(s)$ and $p_{2}(s) \rightarrow 0$ as slowly as we like, we obtain

$$
\int_{4+\frac{64+p_{1}(s)}{s-16}}^{4+\frac{64+p_{2}(s)}{s-16}} \rho(s, t) d t \geq \frac{p_{2}(s)-p_{1}(s)}{36 \pi s^{3 / 2}}\left(\frac{p_{1}(s)}{32}\right)^{3} a_{0}^{4} .
$$

This bound is of interest as it shows that the asymptotic inelastic cross section cannot vanish if the $S$-wave scattering length is nonzero. However, the bound (50) is preferable as it does not need any asymptotic approximation.

\section{ASYMPTOTIC BEHAVIOR OF THE LOWER BOUND ON THE INELASTIC CROSS SECTION, AND DISCUSSION OF THE ASSUMPTIONS}

Now we know that, above a certain energy, the inelastic cross section cannot be zero. A lower bound can be obtained if we know something about $B(s)$ and if we accept the postulated analyticity. If we believe in the validity of the Mandelstam representation with a finite number of subtractions, then $B(s)=s^{N}$. In fact, we tend to believe that $B(s)=s^{2} / s_{0}^{2}$, because we postulate an ellipse (with cuts) which in the limit of high energy coincides with the ellipse with foci $t=0, u=0$ and extremities $t=4$, $u=4$. Inside this ellipse the absorptive part $F_{s}(s, t)$ is maximum for real $t, 0<t<4$, and the integral

$$
\int \frac{F_{s}(s, t) d s}{s^{3}}<\infty
$$

which means that $F_{s}(s, t)$ is almost everywhere less than $s^{2}$. Concerning the dispersive part-which is, modulo subtractions, the Hilbert transform of the absorptive part-we have a rather tricky argument to show again that it is almost everywhere bounded by $s^{2+\epsilon}$, with $\epsilon$ arbitrarily small, for any $t$ for which dispersion relations are valid. But we shall not use that result here.

Using the lower bound on the integral of the double spectral function, and $B(s)=s^{N}$, we deduce that the ratio of the contributions of the cut term and the elliptical contour $(\Gamma)$ term to $\operatorname{Im} f_{l}$ goes to infinity if

$$
l>L_{0}(s)=\frac{(N+5 / 2)}{16} s \ln s .
$$

The ratio of the contribution of the cut term to $\operatorname{Im} f_{l}$ to the upper bound on $\left|f_{l}\right|^{2}$ goes to infinity for a much smaller value, viz., if

$$
l>L_{1}(s)=\frac{\sqrt{s}}{4}(2 N+5 / 2) \ln s .
$$

Hence, summing the contributions of partial waves with $l>L_{0}(s)$, we see that for $s \rightarrow \infty$

$$
\sigma_{\text {inel }}(s)>\frac{\text { const }}{s^{5 / 2}} \exp \left[-\frac{\sqrt{s}}{4}(N+5 / 2) \ln s\right] .
$$

\section{REAL PIONS OF ISOTOPIC SPIN 1}

Let $F^{(I)}(s, t, u)$ denote the $\pi \pi \rightarrow \pi \pi$ amplitudes with total isospin $I$ in the $s$ channel, $I=0,1,2$, and $F^{(I)}(t, s, u)$ the amplitudes with isospin $I$ in the $t$ channel. They are related by the crossing matrix $C_{s t}$,

$$
\begin{aligned}
{\left[\begin{array}{l}
F^{(0)}(t, s, u) \\
F^{(1)}(t, s, u) \\
F^{(2)}(t, s, u)
\end{array}\right] } & =C_{s t}\left[\begin{array}{l}
F^{(0)}(s, t, u) \\
F^{(1)}(s, t, u) \\
F^{(2)}(s, t, u)
\end{array}\right], \\
C_{s t} & =\left[\begin{array}{ccc}
1 / 3 & 1 & 5 / 3 \\
1 / 3 & 1 / 2 & -5 / 6 \\
1 / 3 & -1 / 2 & 1 / 6
\end{array}\right] .
\end{aligned}
$$

We do not assume the unsubtracted Mandelstam representation,

$$
\begin{aligned}
F^{(I)}(s, t, u)= & \frac{1}{\pi^{2}} \iint \frac{\rho_{s t}^{(I)}\left(s^{\prime}, t^{\prime}\right) d s^{\prime} d t^{\prime}}{\left(s^{\prime}-s\right)\left(t^{\prime}-t\right)} \\
& +\frac{1}{\pi^{2}} \iint \frac{\rho_{s u}^{(I)}\left(s^{\prime}, u^{\prime}\right) d s^{\prime} d u^{\prime}}{\left(s^{\prime}-s\right)\left(u^{\prime}-u\right)} \\
& +\frac{1}{\pi^{2}} \iint \frac{\rho_{t u}^{(I)}\left(t^{\prime}, u^{\prime}\right) d t^{\prime} d u^{\prime}}{\left(t^{\prime}-t\right)\left(u^{\prime}-u\right)} .
\end{aligned}
$$

However, we use the definitions

$$
\begin{aligned}
& F_{s t}^{(I)}(s, t, u)=\rho_{s t}^{(I)}(s, t), \\
& F_{s u}^{(I)}(s, t, u)=\rho_{s u}^{(I)}(s, u), \\
& F_{t u}^{(I)}(s, t, u)=\rho_{t u}^{(I)}(t, u),
\end{aligned}
$$

and Eq. (59) then implies that

$$
F_{s t}^{(I)}(t, s, u)=\rho_{s t}^{(I)}(t, s)=\sum_{I^{\prime}=0,1,2} C_{s t}^{I I^{\prime}} \rho_{s t}^{\left(I^{\prime}\right)}(s, t) .
$$

Note that in $\rho_{s t}^{(I)}(t, s)$ and $\rho_{s t}^{\left(I^{\prime}\right)}(s, t)$, the superscripts $I, I^{\prime}$ denote isospins in the channel specified by the first 
argument, viz., $t$ channel and $s$ channel, respectively. The Mandelstam unitarity equations for the $t$-channel isospin $I$, and $4 \leq t \leq 16$, is given in Ref. [5],

$$
\begin{aligned}
\rho^{(I)}(t, s) & =\frac{2 \rho(t)}{\pi} \iint \frac{d z_{1} d z_{2} \theta\left(z-z_{+}\right)}{\sqrt{H\left(z, z_{1}, z_{2}\right)}} G^{(I)}\left(t, s_{1}, s_{2}\right), \\
G^{(I)}\left(t, s_{1}, s_{2}\right) & =(-1)^{I} F_{s}^{(I)}\left(t, s_{1}\right) F_{s}^{(I) *}\left(t, s_{2}\right) .
\end{aligned}
$$

Crossing, Eq. (59) immediately yields

$$
\begin{aligned}
G^{(I)}\left(t, s_{1}, s_{2}\right) & =\sum_{I^{\prime}, I^{\prime \prime}=0,1,2} \zeta_{I^{\prime} I^{\prime \prime}}^{I} F_{s}^{\left(I^{\prime}\right)}\left(s_{1}, t\right) F_{s}^{\left(I^{\prime \prime}\right)}\left(s_{2}, t\right)^{*}, \\
\zeta_{I^{\prime} I^{\prime \prime}}^{I} & =(-1)^{I} C_{s t}^{I I^{\prime}} C_{s t}^{I I^{\prime \prime}},
\end{aligned}
$$

where

$$
\begin{aligned}
\zeta^{0} & =\left[\begin{array}{ccc}
1 / 9 & 1 / 3 & 5 / 9 \\
1 / 3 & 1 & 5 / 3 \\
5 / 9 & 5 / 3 & 25 / 9
\end{array}\right], \\
\zeta^{1} & =\left[\begin{array}{ccc}
-1 / 9 & -1 / 6 & 5 / 18 \\
-1 / 6 & -1 / 4 & 5 / 12 \\
5 / 18 & 5 / 12 & -25 / 36
\end{array}\right], \\
\zeta^{2} & =\left[\begin{array}{ccc}
1 / 9 & -1 / 6 & 1 / 18 \\
-1 / 6 & 1 / 4 & -1 / 12 \\
1 / 18 & -1 / 12 & 1 / 36
\end{array}\right],
\end{aligned}
$$

which are identical to the values obtained in Ref. [5] and we quote them again for reference. We now have

$$
\begin{aligned}
\rho^{(I)}(t, s)= & \frac{2 \rho(t)}{\pi} \iint \frac{d z_{1} d z_{2} \theta\left(z-z_{+}\right)}{\sqrt{H\left(z, z_{1}, z_{2}\right)}} \\
& \times \sum_{I^{\prime}, I^{\prime \prime}=0,1,2} \zeta_{I^{\prime} I^{\prime \prime}}^{I} F_{s}^{\left(I^{\prime}\right)}\left(s_{1}, t\right) F_{s}^{\left(I^{\prime \prime}\right)}\left(s_{2}, t\right)^{*} .
\end{aligned}
$$

Mahoux and Martin [5] have noted that all of the matrix elements of

$$
\zeta^{0}, \zeta^{0}-\zeta^{2}, \zeta^{0}+\zeta^{1}, \zeta^{0}-2 \zeta^{1}, \text { and } \zeta^{0}+2 \zeta^{2}
$$

are positive, and for $s, t$ in the Mahoux-Martin domain (15) the $F_{s}^{(I)}\left(s_{i}, t\right), i=1,2$ are positive for the relevant values of $s_{i}$ due to unitarity. From Eq. (66), it follows that

$\sum_{I} \beta_{I} \zeta_{I^{\prime}, I^{\prime \prime}}^{I}>0, \quad$ for all $I^{\prime}, I^{\prime \prime} \Rightarrow \sum_{I} \beta_{I} \rho^{(I)}(t, s)>0$. $\rho^{(0)}(t, s), \rho^{(0)}(t, s)-\rho^{(2)}(t, s), \rho^{(0)}(t, s)+\rho^{(1)}(t, s)$,

$\rho^{(0)}(t, s)-2 \rho^{(1)}(t, s)$, and $\rho^{(0)}(t, s)+2 \rho^{(2)}(t, s)$

are positive in the Mahoux-Martin domain. We can exploit these results to get bounds on inelastic cross sections for real pions (of isospin 1).

\section{A. New results}

The truncated Froissart-Gribov formula will enable us to obtain lower bounds on imaginary parts of $s$-channel partial waves of the following five amplitudes:

$$
\begin{aligned}
\left(\frac{1}{3} F^{(0)}+F^{(1)}+\frac{5}{3} F^{(2)}\right)(s, t) & =F^{(0)}(t, s), \\
\frac{3}{2}\left(F^{(1)}+F^{(2)}\right)(s, t) & =\left(F^{(0)}-F^{(2)}\right)(t, s), \\
\left(\frac{2}{3} F^{(0)}+\frac{3}{2} F^{(1)}+\frac{5}{6} F^{(2)}\right)(s, t) & =\left(F^{(0)}+F^{(1)}\right)(t, s), \\
\left(-\frac{1}{3} F^{(0)}+\frac{10}{3} F^{(2)}\right)(s, t) & =\left(F^{(0)}-2 F^{(1)}\right)(t, s), \\
\frac{1}{3}\left(F^{(0)}+2 F^{(2)}\right)(s, t) & =\frac{1}{3}\left(F^{(0)}+2 F^{(2)}\right)(t, s),
\end{aligned}
$$

where the right-hand sides correspond to the $t$-channel isospin combinations in Eq. (69), and the left-hand sides are the corresponding linear combinations of $s$-channel isospin amplitudes. These equations are of the form

$$
\sum_{I} \alpha_{I} F^{(I)}(s, t)=\sum_{I} \beta_{I} F^{(I)}(t, s),
$$

where the coefficients $\alpha_{I}$ and $\beta_{I}$ can be read from Eq. (70). E.g., $\alpha_{0}=\beta_{0}=1 / 3, \alpha_{2}=\beta_{2}=2 / 3, \alpha_{1}=\beta_{1}=0$ for the last amplitude, which is just the $\pi^{0} \pi^{0} \rightarrow \pi^{0} \pi^{0}$ amplitude,

$$
F^{00} \equiv \frac{1}{3}\left(F^{(0)}+2 F^{(2)}\right) .
$$

The partial waves given by the truncated Froissart-Gribov formula are then, for even $l+I$,

$$
\begin{aligned}
\sum_{I} \alpha_{I} f_{l}^{I}(s) & \\
= & \frac{1}{4 i \pi k^{2}} \int_{\Gamma} Q_{l}\left(1+\frac{2 t}{s-4}\right) \sum_{I} \beta_{I} F^{(I)}(t, s) d t \\
& +\frac{1}{\pi k^{2}} \int_{4}^{4+\frac{32}{\sqrt{s}-6}} Q_{l}\left(1+\frac{2 t}{s-4}\right) \sum_{I} \beta_{I} F_{t}^{(I)}(t, s) d t
\end{aligned}
$$

and 


$$
\begin{aligned}
\sum_{I} \alpha_{I} \operatorname{Im} f_{l}^{I}(s) & \\
= & \frac{1}{4 i \pi k^{2}} \int_{\Gamma} Q_{l}\left(1+\frac{2 t}{s-4}\right) \sum_{I} \beta_{I} F_{s}^{(I)}(t, s) d t \\
& +\frac{1}{\pi k^{2}} \int_{4+\frac{64}{s-4}}^{4+\frac{32}{\sqrt{s}-6}} Q_{l}\left(1+\frac{2 t}{s-4}\right) \sum_{I} \beta_{I} \rho^{(I)}(t, s) d t .
\end{aligned}
$$

As before, $\Gamma$ is an ellipse with foci at $t=0$ and $u=0$, and right extremity at $t=4+\frac{32}{\sqrt{s}-6}$. As for pions without isospin, if we only use the region $A$ in Fig. (2), we can prove that the combinations $\sum_{I} \beta_{I} \rho^{(I)}(t, s)$ on the righthand side are not only positive, but also have a lower bound,

$\sum_{I} \beta_{I} \rho^{(I)}(t, s) \geq \frac{4}{\pi s \sqrt{t(t-4)}} \sum_{I} \beta_{I} \zeta_{I^{\prime}, I^{\prime \prime}}^{I} I^{I^{\prime}}\left(s_{M}\right) I^{I^{\prime \prime}}\left(s_{M}\right)$

provided that $\sum_{I} \beta_{I} \zeta_{I^{\prime}, I^{\prime \prime}}^{I}>0$, for all $I^{\prime}, I^{\prime \prime}$, and

$$
I^{I^{\prime}}\left(s_{M}\right) \equiv \int_{4}^{s_{M}} \frac{d s_{1} k_{1} \sqrt{s_{1}} \sigma_{\mathrm{tot}}^{\left(I^{\prime}\right)}\left(s_{1}\right)}{16 \pi}
$$

We can now obtain lower bounds on the cut contributions to linear combinations of imaginary parts of $s$-channel partial waves,

$$
\begin{array}{ll}
1 / 3 f_{l}^{(0)}+f_{l}^{(1)}+5 / 3 f_{l}^{(2)}, & 3 / 2\left(f_{l}^{(1)}+f_{l}^{(2)}\right), \\
2 / 3 f_{l}^{(0)}+3 / 2 f_{l}^{(1)}+5 / 6 f_{l}^{(2)}, & -1 / 3 f_{l}^{(0)}+10 / 3 f_{l}^{(2)}, \\
1 / 3 f_{l}^{(0)}+2 / 3 f_{l}^{(2)}
\end{array}
$$

from lower bounds, respectively, on the combinations of $\rho^{(I)}(t, s)$ given in Eq. (69). The contributions to these imaginary parts from the elliptical contours $\Gamma$ are negligible for $l>L_{0}(s)$; the elastic pion-pion cross sections (including $\pi^{0} \pi^{0} \rightarrow \pi^{+} \pi^{-}$cross sections) are negligible for $l>L_{1}(s)$, and hence also for $l>L_{0}(s)$. On summing the contributions of $l>L_{0}(s)$ lower bounds on $\operatorname{Im}\left[3 / 2\left(f_{l}^{(1)}+f_{l}^{(2)}\right)\right]$ and $\operatorname{Im}\left[1 / 3 f_{l}^{(0)}+2 / 3 f_{l}^{(2)}\right]$ to inelastic cross sections, we obtain the three inequalities

$$
\begin{aligned}
& \sigma_{\text {inel }}^{(1)}(s), \sigma_{\text {inel }}^{(2)}(s), \sigma_{\text {inel }}^{\pi^{0} \pi^{0}}(s) \\
& \quad>\frac{\text { const }}{s^{5 / 2}} \exp \left[-\frac{\sqrt{s}}{4}(N+5 / 2) \ln s\right] .
\end{aligned}
$$

\section{ACKNOWLEDGMENTS}

A. M. is grateful to João Penedones for inviting him to participate in a workshop on S-matrix bootstrap in Lausanne, and to Miguel F. Paulos for raising the question whether inelasticity could be arbitrarily small. S. M. R. wishes to thank the Indian National Science Academy for the INSA Honorary Scientist position.

\section{APPENDIX: BOUNDS ON ASSOCIATED LEGENDRE FUNCTIONS}

We derive bounds on $Q_{l}(x)$ for real $l$ and complex $x$ using the integral representation,

$$
Q_{l}(x)=\int_{0}^{\infty} \frac{d t}{\left(x+\sqrt{x^{2}-1} \cosh t\right)^{l+1}} .
$$

\section{Upper bound}

For real $x>1$,

$$
Q_{l}(x) \leq\left(x+\sqrt{x^{2}-1}\right)^{-l} Q_{0}(x) .
$$

This is obvious because $x+\sqrt{x^{2}-1} \cosh t \geq\left(x+\sqrt{x^{2}-1}\right)$.

\section{Lower bound}

For real $x>1$,

$$
Q_{l}(x) \geq\left(x+\sqrt{x^{2}-1}\right)^{-l-1} \sqrt{\frac{\pi}{2(l+1)}} .
$$

Proof: It is obvious that

$$
Q_{l}(x) \geq\left(x+\sqrt{x^{2}-1}\right)^{-l-1} \int_{0}^{\infty} \frac{d t}{(\cosh t)^{l+1}},
$$

because $\left(x+\sqrt{x^{2}-1} \cosh t\right) \leq\left(x+\sqrt{x^{2}-1}\right) \cosh t$. The integral on the right-hand side is exactly known [6],

$$
\int_{0}^{\infty} \frac{d t}{(\cosh t)^{l+1}}=\frac{2^{l-1}}{\Gamma(l+1)} \Gamma^{2}\left(\frac{l+1}{2}\right),
$$

but we shall only need a lower bound on it. Using $\cosh t \leq \exp \left(t^{2} / 2\right)$, we have

$$
\begin{aligned}
\int_{0}^{\infty} \frac{d t}{(\cosh t)^{l+1}} & \geq \int_{0}^{\infty} d t \exp \left(-t^{2}(l+1) / 2\right) \\
& =\sqrt{\frac{\pi}{2(l+1)}}
\end{aligned}
$$

Inserting this into Eq. (A4), we obtain the quoted lower bound (A3). 


\section{Upper bound on an ellipse in the complex $z$ plane}

We prove that for real values of $\theta_{1}, \theta_{2}$,

$$
\left|Q_{l}\left(\cosh \left(\theta_{1}+i \theta_{2}\right)\right)\right| \leq Q_{l}\left(\cosh \theta_{1}\right),
$$

i.e., geometrically, for $z$ on an ellipse with foci -1 and 1 and right extremity $z_{0}=\cosh \theta_{1}$,

$$
\left|Q_{l}(z)\right| \leq Q_{l}\left(z_{0}\right) \quad \text { for } z=\cosh \left(\theta_{1}+i \theta_{2}\right) .
$$

The denominator in the integral representation of $Q_{l}(z)$ is $|D(z, t)|^{l+1}$, where

$D(z, t)=\cosh \left(\theta_{1}+i \theta_{2}\right)+\cosh t \sinh \left(\theta_{1}+i \theta_{2}\right)$.

It suffices to prove that

$$
|D(z, t)|>\left.D(z, t)\right|_{\theta_{2}=0} .
$$

Trigonometric identities yield

$$
\begin{aligned}
|D(z, t)|^{2}= & D(z, t) D(z, t)^{*}=\frac{1}{2} \cosh 2 \theta_{1}\left(1+\cosh ^{2} t\right) \\
& +\cosh t \sinh 2 \theta_{1}-\frac{1}{2} \cos 2 \theta_{2} \sinh ^{2} t .
\end{aligned}
$$

Minimizing over $\theta_{2}$ now yields the desired result, Eq. (A10).

\section{Upper bound on $Q_{l}(x)$ in terms of $Q_{0}(x)$ and $Q_{l}\left(2 x^{2}-1\right)$ for $x>1$}

We prove that

$$
Q_{l}^{2}(x) \leq 2 x Q_{0}(x) Q_{l}\left(2 x^{2}-1\right), \quad \text { for } x>1 .
$$

(i) The integral representation of $Q_{l}(x)$ and the Schwarz inequality yield

$$
Q_{l}^{2}(x) \leq Q_{0}(x) Q_{2 l}(x)
$$

Hence, to prove Eq. (A12) it will be sufficient to prove that

$$
Q_{2 l}(x) \leq 2 x Q_{l}\left(2 x^{2}-1\right) .
$$

Using

$$
\begin{aligned}
(x+ & \left.\sqrt{x^{2}-1} \cosh t\right)^{2}=2 x^{2}-1 \\
& +\sqrt{\left(2 x^{2}-1\right)^{2}-1} \cosh t+\left(x^{2}-1\right) \sinh ^{2} t \\
\geq & 2 x^{2}-1+\sqrt{\left(2 x^{2}-1\right)^{2}-1} \cosh t,
\end{aligned}
$$

and

$$
\begin{array}{r}
2 x^{2}-1+\sqrt{\left(2 x^{2}-1\right)^{2}-1} \cosh t \\
=2 x\left(x+\sqrt{x^{2}-1} \cosh t\right)-1,
\end{array}
$$

we have the required result

$$
\begin{aligned}
Q_{2 l}(x) \leq & \int_{0}^{\infty} d t\left(2 x-\frac{1}{x+\sqrt{x^{2}-1} \cosh t}\right) \\
& \times \frac{1}{\left(2 x^{2}-1+\sqrt{\left(2 x^{2}-1\right)^{2}-1} \cosh t\right)^{l+1}} \\
\leq & 2 x Q_{l}\left(2 x^{2}-1\right) .
\end{aligned}
$$

\section{Upper bound on $Q_{l}(x) / Q_{l}(z)$ \\ for $x>z>1$}

We prove that for $x>z>1$

$\frac{Q_{l}(x)}{Q_{l}(z)} \leq\left(\frac{z+\sqrt{z^{2}-1}}{x+\sqrt{x^{2}-1}}\right)^{l+1} \leq\left(\frac{1+\sqrt{2(z-1)}}{1+\sqrt{2(x-1)}}\right)^{l+1}$.

Using the integral representation, we obtain

$$
\begin{aligned}
\frac{d}{d z}( & \left.\left(z+\sqrt{z^{2}-1}\right)^{l+1} Q_{l}(z)\right) \\
= & -\frac{l+1}{\sqrt{z^{2}-1}} \times\left(z+\sqrt{z^{2}-1}\right)^{l} \\
& \times \int_{0}^{\infty} \frac{d t(\cosh t-1)}{\left(z+\sqrt{z^{2}-1} \cosh t\right)^{l+2}} \\
\leq & 0,
\end{aligned}
$$

which implies the left-hand side of the inequality (A18). The right-hand side now follows if

$$
\left(\frac{z+\sqrt{z^{2}-1}}{x+\sqrt{x^{2}-1}}\right) \leq\left(\frac{1+\sqrt{2(z-1)}}{1+\sqrt{2(x-1)}}\right),
$$

or if

$$
\left(\frac{z+\sqrt{z^{2}-1}}{1+\sqrt{2(z-1)}}\right) \leq\left(\frac{x+\sqrt{x^{2}-1}}{1+\sqrt{2(x-1)}}\right)
$$

for $x>z>1$. This holds since the left-hand side of the above inequality is an increasing function of $z$ for $z>1$. 
[1] F. F. K. Cheung and J. S. Toll, Phys. Rev. 160, 1072 (1967).

[2] A. Martin, Nuovo Cimento 42A, 930 (1966); 44A, 1219 (1966).

[3] A. J. Dragt, Phys. Rev. 156, 1588 (1967).

[4] S. Mandelstam, Phys. Rev. 112, 1344 (1958).
[5] G. Mahoux and A. Martin, Nuovo Cimento 33, 883 (1964).

[6] I. S. Gradshteyn and I. M. Ryzhik, Tables of Integrals, Series, and Products (Academic Press, Cambridge, MA, 1980), p. 506. 